\title{
Imaging-based definition of lower uterine segment carcinoma to improve the detection sensitivity of probable Lynch syndrome
}

\author{
Hidenori Kato, Hiroyuki Yamazaki, Yukiharu Todo, Hiroko Matsumiya, \\ Shinichiro Minobe, Tomohiko Tsuruta
}

\section{Division of Gynecologic Oncology, National Hospital Organization, Hokkaido Cancer Center, 4-2 Kikusui, Shiroishi-ku, Sapporo, Hokkaido, 003-0804, Japan}

Objective: The aim of this study was to investigate a magnetic resonance imaging (MRI)-based definition of lower uterine segment (LUS) carcinoma.

Methods: We retrospectively reviewed 587 consecutive patients with endometrial cancer who underwent hysterectomy. LUS carcinoma was determined through pathological examination and MRI assessment. For imaging assessment, the location of the inner lining of the uterus was classified into four equal parts on a sagittal section image(Fig.1). A tumor was defined as LUS carcinoma when its thickest part was located in the second or the third part from the uterine fundus. LUS carcinoma was further divided into LUS in a narrow sense, upon which diagnosis was exclusively based on pathological findings. The relationship between LUS carcinoma and probable Lynch Syndrome (LS) was investigated. Patients with loss of MSH2, MSH6, and PMS2 expression or those with tumors with loss of MLH1 and absence of $M L H 1$ promoter methylation were diagnosed as probable $L S$.

Results: LUS carcinoma was identified in 59 (10.2\%) patients. Twenty-eight $(47.5 \%)$ patients were categorized as LUS in a narrow sense and $31 \mathbf{( 5 2 . 5 \% )}$ as LUS in a broad sense. Among them, probable LS was identified in $12(20.3 \%)$ cases (Table1). There was no difference in clinical profiles (Table2), including the prevalence of probable LS between the two categories.

Conclusions: An MRI-based, expanded definition of LUS carcinoma is likely to secure characteristics equivalent to a conventional, pathology-based definition of LUS carcinoma. The novel definition of LUS carcinoma might improve the detection of probable LS.

MMR protein expression and MLH1 promoter methylation

analysis

sporadic pattern

MLH1/PMS2 unexpression

(MLH1 unmethylated)

(MLH1 methylated)

(methylation analysis defect)

MSH2/MSH6 unexpression

MSH6 unexpression

PMS2 unexpression

MSH2/PMS2 unexpression
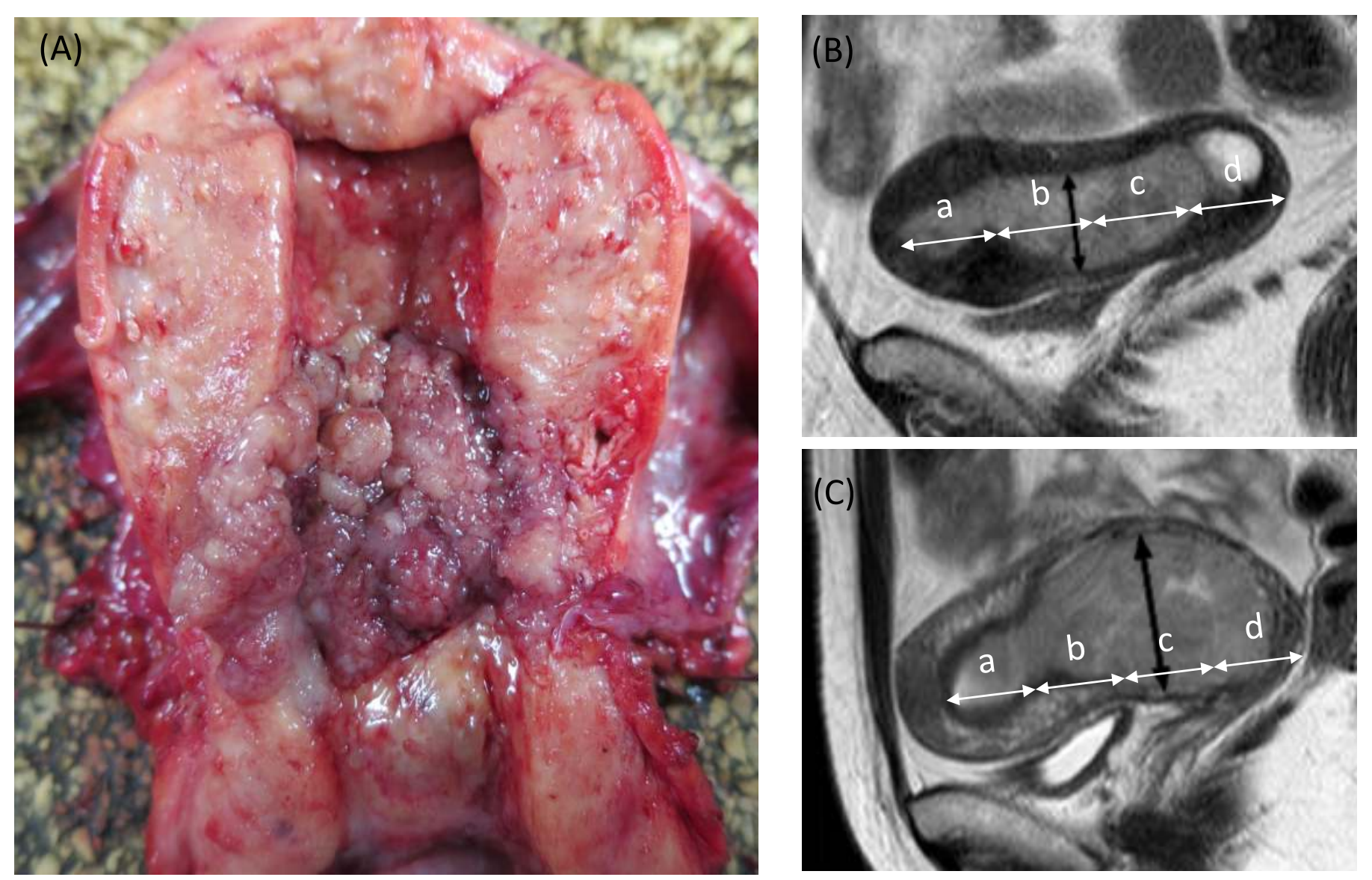

Figure 1. LUS carcinoma in a narrow sense; (A) Tumor exclusively present in the lower uterine segment on gross/pathological inspection. LUS carcinoma in a broad sense; The thickest part of a tumor is identified in the second (B) or third (C) part and another part is present in the first and/or fourth part from the uterine fundus on a MRI-based sagittal section image.

\begin{tabular}{|c|c|c|c|}
\hline & $\begin{array}{l}\text { LUS in a narrow } \\
\text { sense }\end{array}$ & LUS in a broad sense & $p$-value \\
\hline & $(n=28)$ & $(n=31)$ & \\
\hline Age & mean 58.3yo & mean 53.4 yo & 0.12 \\
\hline BMI & mean 25.2 & mean 25.3 & 0.98 \\
\hline \multicolumn{4}{|l|}{$\begin{array}{l}\text { FIGO stage } \\
\text { (2008) }\end{array}$} \\
\hline 1 & $16(57.1 \%)$ & $11(35.5 \%)$ & 0.38 \\
\hline II & $5(17.9 \%)$ & $8(25.8 \%)$ & \\
\hline III & $6(21.4 \%)$ & $9(29.0 \%)$ & \\
\hline IV & $1(3.6 \%)$ & $3(9.7 \%)$ & \\
\hline \multicolumn{4}{|l|}{$\begin{array}{l}\text { Tumor grade/ } \\
\text { histology }\end{array}$} \\
\hline Endometrioid & & & 0.93 \\
\hline G1 & $12(42.9 \%)$ & $13(41.9 \%)$ & \\
\hline $\mathrm{G} 2$ & $4(14.3 \%)$ & $6(19.4 \%)$ & \\
\hline G3 & $7(25.0 \%)$ & $8(25.8 \%)$ & \\
\hline $\begin{array}{l}\text { Non- } \\
\text { endometrioid }\end{array}$ & $5(17.9 \%)$ & $4(12.9 \%)$ & \\
\hline \multicolumn{4}{|l|}{$\begin{array}{l}\text { Myometrial } \\
\text { invasion }\end{array}$} \\
\hline$<1 / 2$ & $15(53.6 \%)$ & $13(41.9 \%)$ & 0.37 \\
\hline$>1 / 2$ & $13(46.4 \%)$ & $18(58.1 \%)$ & \\
\hline MMR analysis & & & 0.84 \\
\hline sporadic & $23(82.1 \%)$ & $25(80.6 \%)$ & \\
\hline probable LS & $6(21,4 \%)$ & $6(19.4 \%)$ & \\
\hline
\end{tabular}

LUS lower uterine segment carcinoma, BMI body mass index, FIGO the International Federation of Gynecology and Obstetrics, MMR mismatch repair, LS Lynch syndrome

Table 2. Clinical characteristics of patients with lower uterine segment (LUS) carcinoma by narrow and broad definitions of LUS 